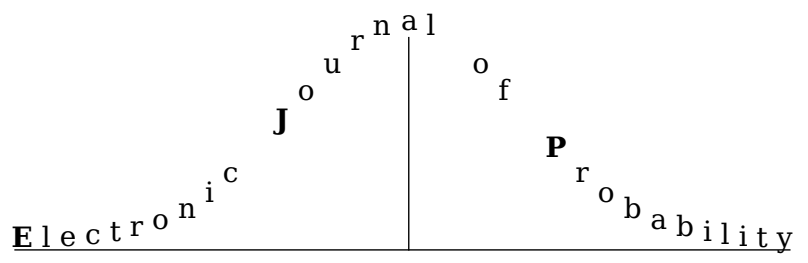

Electron. J. Probab. 25 (2020), article no. 94, 1-22.

ISSN: 1083-6489 https://doi.org/10.1214/20-EJP495

\title{
Functional inequalities for forward and backward diffusions*
}

\author{
Daniel Bartl ${ }^{\dagger} \quad$ Ludovic Tangpi ${ }^{\ddagger}$
}

\begin{abstract}
In this article we derive Talagrand's $T_{2}$ inequality on the path space w.r.t. the maximum norm for various stochastic processes, including solutions of one-dimensional stochastic differential equations with measurable drifts, backward stochastic differential equations, and the value process of optimal stopping problems.

The proofs do not make use of the Girsanov method, but of pathwise arguments. These are used to show that all our processes of interest are Lipschitz transformations of processes which are known to satisfy desired functional inequalities.
\end{abstract}

Keywords: quadratic transportation inequality; optimal stopping; backward stochastic differential equation; stochastic differential equation; non-smooth coefficients; concentration of measures; logarithmic-Sobolev inequality.

MSC2020 subject classifications: 60J60; 60G40; 28C20; 60E15; 60H20; 91G10.

Submitted to EJP on October 3, 2019, final version accepted on July 4, 2020.

Supersedes arXiv: 1910.00504.

\section{Introduction and main results}

\subsection{Notation}

Let $\left(\Omega, \mathcal{F},\left(\mathcal{F}_{t}\right), P\right)$ be the canonical space of a $d$-dimensional Brownian motion $W$ equipped with the $P$-completion of the filtration $\sigma\left(W_{s}: s \leq t\right)$ generated by $W$. That is, $\Omega=C\left([0, T], \mathbb{R}^{d}\right)$ is endowed with the maximum norm, $W_{t}(\omega)=\omega(t)$, and $P$ is the Wiener measure. For $p \in[1, \infty)$ and $\mu, \nu \in \mathcal{P}(\Omega)$ (the set of all Borel probability on $\Omega$ ) define the $p$-Wasserstein distance and the relative entropy by

$$
\mathcal{W}_{p}(\mu, \nu):=\left(\inf _{\pi} \int_{\Omega \times \Omega}\|\omega-\eta\|_{\infty}^{p} \pi(d \omega, d \eta)\right)^{1 / p} \quad \text { and } \quad H(\nu \mid \mu):=\int_{\Omega} \frac{d \nu}{d \mu} \log \frac{d \nu}{d \mu} d \mu
$$

where the infimum is taken over all couplings $\pi$ (that is, probability measures on the product with first marginal $\mu$ and second marginal $\nu$ ) and we used the convention $d \nu / d \mu=$

\footnotetext{
*Daniel Bartl is supported by the Austrian Science Fund (FWF) under project P28661 and by the Vienna Science and Technology Fund (WWTF) under project MA16-021; Ludovic Tangpi is supported by the NSF grant DMS-2005832. Both authors thank an anonymous referee and an associate editor for their comments.

${ }^{\dagger}$ University of Vienna, Austria. E-mail: daniel . bartl@univie.ac .at

${ }^{\ddagger}$ Princeton University, United States of America. E-mail: ludovic.tangpi@princeton. edu
} 
$+\infty$ if $\nu$ is not absolutely continuous w.r.t. $\mu$. Recall that the quadratic transportation inequality (sometimes called Talagrand's inequality) reads as

$$
\mu \text { satisfies } T_{2}(C) \text { if } \mathcal{W}_{2}(\mu, \nu) \leq \sqrt{C H(\nu \mid \mu)} \text { for all } \nu \in \mathcal{P}(\Omega) .
$$

The validity of this inequality has several (deep) consequences, for instance for the concentration of measure phenomenon, the isoperimetric problem and various problems of probability in high dimensions. We refer the reader e.g. to [36, 53, 49, 37] for an overview and applications. Let us for instance mention a result by Gozlan, see [26, Theorem 1.3], who showed that $T_{2}(C)$ is equivalent to the dimension-free concentration

$$
\mu^{n}\left(\left|F-\int F d \mu^{n}\right|>x\right) \leq 2 \exp \left(-c x^{2}\right)
$$

for all $x>0, n \geq 1$ and some constant $c>0$, where $\mu^{n}$ is the $n$-fold product of $\mu$ and $F: \Omega^{n} \rightarrow \mathbb{R}$ is a 1-Lipschitz function w.r.t. the $l_{2}$-norm on $\Omega^{n}$.

\subsection{Main results}

In this work, we prove the validity of $T_{2}$ for various stochastic processes evolving forward and backward in time. Let us present our principal contribution; the proofs are postponed to later sections along with applications and consequences.

\subsubsection{Optimal stopping}

Our first result concerns the value process of an optimal stopping problem.

Theorem 1.1 (Optimal Stopping). Let $\Gamma:[0, T] \times \Omega \rightarrow \mathbb{R}$ be an adapted process with continuous paths such that $\Gamma_{t}$ is $L_{\Gamma}$-Lipschitz for every $t \in[0, T]$ and denote by

$$
S_{t}:=\operatorname{ess.sup}_{\tau \text { is stopping time, } t \leq \tau \leq T} E\left[\Gamma_{\tau} \mid \mathcal{F}_{t}\right]
$$

(OptStop)

for $t \in[0, T]$ the value process of the optimal stopping problem of $\Gamma$. Then $S$ has continuous paths and

$$
\text { the law } \mu^{s} \text { of } S \text { satisfies } T_{2}\left(C_{s}\right)
$$

with $C_{s}:=2 L_{\Gamma}^{2}$.

A generalization of Theorem 1.1 is given in Corollary 2.5. In many applications it is interesting to approximate the law $\mu^{s}$ of $S$. Denote by $\mu_{N}:=\mu_{N}^{s}$ the empirical measure associated to $\mu:=\mu^{s}$, that is, we fix $P^{\infty}$ the infinite product of $P$ under which i.i.d. random variables $\left(S^{n}\right)_{n \in \mathbb{N}}$ with distribution $\mu$ are defined, and we put $\mu_{N}:=\frac{1}{N} \sum_{n=1}^{N} \delta_{S^{n}}$ for every $N \geq 1$. Applying (1.1) to the 1-Lipschitz function $F(y):=\sqrt{N} \mathcal{W}_{2}\left(\frac{1}{N} \sum_{n=1}^{N} \delta_{y^{n}}, \mu\right)$ gives the following concentration property of Wasserstein distance between the true and the empirical measure:

Corollary 1.2. In the setting of Theorem 1.1 there is $c>0$ such that

$$
P^{\infty}\left(\left|\mathcal{W}_{2}\left(\mu, \mu_{N}\right)-E_{P \infty}\left[\mathcal{W}_{2}\left(\mu, \mu_{N}\right)\right]\right| \geq x\right) \leq 2 \exp \left(-c x^{2} N\right)
$$

for every $x>0$ and $N \geq 1$.

Also note that by convergence of $E_{P \infty}\left[\mathcal{W}_{2}\left(\mu, \mu_{N}\right)\right]$ to zero, the above implies that there is $c>0$ and, for every $x>0$, some $N_{0}(x)$ such that

$$
P^{\infty}\left(\mathcal{W}_{2}\left(\mu, \mu_{N}\right) \geq x\right) \leq 2 \exp \left(-c x^{2} N\right)
$$

for all $N \geq N_{0}(x)$. 
Another consequence (which could also be shown by simpler methods) is due to the fact that the $T_{2}$-inequality implies Gaussian concentration [53, Theorem 22.10], that is, Theorem 1.1 in particular implies that

$$
P\left(\left|S_{t}-E\left[S_{t}\right]\right| \geq x\right) \leq 2 \exp \left(-c x^{2}\right)
$$

for all $x>0$, where $c>0$ is a constant. This means that (on a large scale) $E\left[S_{t}\right]$ can be seen as a good proxy for the value of $S_{t}$. This is interesting in that $E\left[S_{t}\right]=\sup _{t \leq \tau \leq T} E\left[\Gamma_{\tau}\right]$ can usually be computed quite efficiently (see e.g. [4]) while the computation of $S_{t}$ might be hard.

\subsubsection{Backward diffusions}

We now turn our attention to the backward stochastic differential equation

$$
Y_{t}=F+\int_{t}^{T} g_{u}\left(Y_{u}, Z_{u}\right) d u-\int_{t}^{T} Z_{u} d W_{u} \quad \text { for } t \in[0, T]
$$

whose solution is given by a pair of processes $(Y, Z)$ on the canonical space $\Omega$ with $Y$ adapted and $Z$ progressive. We have the following:

Theorem 1.3 ( $T_{2}$ for multi-dim BSDE). Let $m \in \mathbb{N}$ and assume that

(A) $g:[0, T] \times \Omega \times \mathbb{R}^{m} \times \mathbb{R}^{m \times d} \rightarrow \mathbb{R}^{m}$ is progressive, $g_{t}(\cdot, \cdot, \cdot)$ is $L_{g}$-Lipschitz continuous for every $t \in[0, T]$, and $E\left[\int_{0}^{T}\left|g_{t}(\cdot, 0,0)\right|^{2} d t\right]<\infty$,

(B) $F: \Omega \rightarrow \mathbb{R}^{m}$ is $L_{F}$-Lipschitz continuous.

Then there exists a unique solution $(Y, Z)$ of (BSDE) and

$$
\text { the law } \mu^{y} \text { of } Y \text { satisfies } T_{2}\left(C_{y}\right)
$$

with $C_{y}:=2\left(L_{F}+T L_{g}\right)^{2} e^{2 T L_{g}}$.

Remarkably, the constant $C_{y}$ in Theorem 1.3 does not depend on $m$ and $d$, suggesting that the result can be extended to infinite dimensional BSDEs (e.g. BSDEs on Hilbert spaces analyzed in [24]). We will not take up this task here. As an (rather direct) application of Theorem 1.3, we derive in Corollary 2.3 a transportation inequality for laws of martingales, thus extending a result by Pal [42]. Moreover, we will show in Section 4 that under additional conditions pertaining to the regularity of $g$, functional inequalities can also be deduced for the law of the control process $Z$.

When $Y$ is one-dimensional (but the Brownian motion still $d$-dimensional) the regularity conditions on $g$ can be weakened as follows:

Theorem 1.4 ( $T_{2}$ for 1-dim BSDE). Assume that

(A) $g:[0, T] \times \mathbb{R}^{d} \rightarrow \mathbb{R}_{+}$is Borel measurable and convex in the last variable,

(B) $g_{t}(z) \leq C\left(1+|z|^{2}\right)$ for all $z \in \mathbb{R}^{d}$ and for some constant $C>0$.

(C) $\inf _{t \in[0, T]} g_{t}(z) /|z| \rightarrow \infty$ as $|z| \rightarrow \infty$, and

(D) $F: \Omega \rightarrow \mathbb{R}$ is bounded from below and $L_{F}$-Lipschitz continuous.

Then (BSDE) admits a unique solution $(Y, Z)$ and

$$
\text { the law } \mu^{y} \text { of } Y \text { satisfies } T_{2}\left(C_{y}\right)
$$

with $C_{y}:=2 L_{F}^{2}$. 
Remark 1.5. When $d=1, g=0$ and $F=\mathrm{id}$, then $Y$ is the Brownian motion, and $C_{y}=2$ (which is known to be optimal for Brownian motion) showing that the constant $C_{y}$ in Theorem 1.3 and Theorem 1.4 cannot be improved in general.

Example 1.6. By Theorem 1.4, the law of the process $Y_{t}:=\log E\left[\exp (F) \mid \mathcal{F}_{t}\right]$ satisfies $T\left(2 L_{F}^{2}\right)$ for every $L_{F}$-Lipschitz continuous function $F$ on $\Omega$ which is bounded from below. In fact, it follows from martingale representation and Itô's formula that there is a progressive process $Z$ such that $(Y, Z)$ solve equation (BSDE) with $g(z)=\frac{1}{2}|z|^{2}$ and terminal condition $F$.

BSDEs provide a powerful probabilistic tool to tackle second order nonlinear partial differential equations as first noted by [45]. They can be seen as a nonlinear generalization of the maximum principle in stochastic control theory. Moreover, BSDEs have various applications in quantitative finance. The following is a prime example stated in more generality (and precision) in Section 3.1.

Example 1.7 (Utility maximization). Let $F: \Omega \rightarrow \mathbb{R}$ be bounded and $L_{F}$-Lipschitz continuous, and consider the Black-Scholes dynamics $d S_{t}=S_{t}\left(d t+d W_{t}\right)$ for the stock price. The process

$$
V_{t}:=\underset{p}{\operatorname{ess} \sup } E\left[U\left(F-\int_{t}^{T} p_{u} \frac{d S_{u}}{S_{u}}\right) \mid \mathcal{F}_{t}\right] \quad \text { with } \quad U(x)=-\exp (-x),
$$

where the supremum is taken over predictable portfolios $p$ subject to some integrability condition, defines the value process of the exponential utility maximization problem in the Black-Scholes market with random endowment $F$.

Then (a suitable transformation of) $V$ and the optimal trading strategy $\pi^{*}$ are characterized by a BSDE and we will see that both satisfy the $T_{2}$-inequality. In particular, concentration of empirical measure as in Corollary 1.2 or Gaussian concentration hold, showing for example that the value of the optimal utility and portfolio are concentrated around their mean.

\subsubsection{Forward diffusions}

Let us finally consider the stochastic differential equation

$$
X_{t}=x+\int_{0}^{t} b_{u}\left(X_{u}\right) d u+\int_{0}^{t} \sigma_{u}\left(X_{u}\right) d W_{u} \quad \text { for } t \in[0, T]
$$

in dimension 1 (again, the Brownian motion is $d$-dimensional).

Theorem 1.8 ( $T_{2}$ for 1-dim SDE). Assume that

(A) $b:[0, T] \times \mathbb{R} \rightarrow \mathbb{R}$ is Borel measurable and continuously differentiable in the first variable,

(B) $\sigma:[0, T] \times \mathbb{R} \rightarrow \mathbb{R}^{d}$ is bounded, continuously differentiable in the first variable and $L_{\sigma}$-Lipschitz continuous in the second variable, and $\sigma \sigma^{\prime} \geq c>0$ for some constant $c$,

(C) the quantities $c_{1}:=\sup _{t \in[0, T]}\left\|\frac{b_{t}}{\sigma_{t} \sigma_{t}^{\prime}}(\cdot)\right\|_{L^{1}(d x)} ; c_{2}:=\sup _{t \in[0, T]}\left\|\frac{b_{t}}{\sigma_{t} \sigma_{t}^{\prime}}(\cdot)\right\|_{L^{\infty}(d x)} ; c_{3}:=$ $\sup _{t \in[0, T]}\left\|\frac{\partial}{\partial t} \frac{b_{t}}{\sigma_{t} \sigma_{t}^{\prime}}(\cdot)\right\|_{L^{1}(d x)}$ and $c_{4}:=\left\|\sup _{t \in[0, T]} \frac{\partial}{\partial t} \frac{b_{t}}{\sigma_{t} \sigma_{t}^{\prime}}(\cdot)\right\|_{L^{1}(d x)}$ are finite.

Then, (SDE) admits a unique strong solution and

$$
\text { the law } \mu^{x} \text { of } X \text { satisfies } T_{2}\left(C_{x}\right)
$$

with $C_{x}:=6 \exp \left(c_{1}+15 \max \left(c_{3} \exp \left(2 c_{1}\right),\|\sigma\|_{\infty} c_{2} \exp \left(2 c_{1}\right)+\exp \left(2 c_{1}\right) L_{\sigma}^{2}\right)\right)$. 
Example 1.9. The above result applies for instance to Langenvin's equations: These are stochastic differential equations of the form

$$
d X_{t}=-U^{\prime}\left(X_{t}\right) d t+\sqrt{2 / \lambda} d W_{t}, \quad X_{0}=x
$$

where $U: \mathbb{R} \rightarrow \mathbb{R}$ is differentiable and plays the role of a potential, and $\lambda>0$ (we take for simplicity $d=1$ ). We will discuss this example further in Section 5.

Remark 1.10. Observe that the statement of Corollary 1.2 remains true for the diffusions considered in Theorem 1.3, Theorem 1.4 or Theorem 1.8.

\subsubsection{Variations and extensions}

The arguments leading to our main results for backwards diffusions and the optimal stopping problem (inspired by techniques used in 'pathwise control theory' see e.g. [19, 40]) consist in showing that the processes under consideration are Lipschitz transforms of Brownian motion. It is likely that this technique also applies to more general stochastic optimal control problems and that different consequences than the above can be deduced.

To illustrate our point, we derive the logarithmic-Sobolev inequality, which reads as:

$$
\mu \in \mathcal{P}\left(\mathbb{R}^{m}\right) \text { satisfies } L S I(C) \text { if } \operatorname{Ent}_{\mu}(f) \leq C \int_{\mathbb{R}^{m}}|\nabla f|^{2} d \mu
$$

for every $\mu$-integrable and differentiable function $f: \mathbb{R}^{m} \rightarrow \mathbb{R}$. Here we denote by $\operatorname{Ent}_{\mu}(f):=\int f^{2} \log \left(f^{2} / \int f^{2} d \mu\right) d \mu$ the entropy of $f$ w.r.t. $\mu$ with the convention $0 / 0=0$.

Theorem 1.11 (Log-Sobolev). In the setting of either Theorem 1.3 or Theorem 1.4, for every $t \in[0, T]$ one has that

$$
\text { the law } \mu_{t}^{y} \text { of } Y_{t} \text { satisfies } \operatorname{LSI}\left(T C_{y}\right)
$$

with the constant $C_{y}$ given in the respective theorems.

The same holds true in the setting of Theorem 1.1 (if $Y$ above is replaced by $S$ ).

\subsection{Related literature}

Measure concentration is a popular area of modern probability theory. This is mostly due to its variety of applications, including (and certainly not restricted to) model selection, random algorithms, quantitative finance and statistics $[38,7,8,34$, 50, 18, 43]. It is the works of Marton [37] and Talagrand [49] that first underlined the relevance of transportation inequalities in the description of the concentration of measure phenomenon. Transportation inequalities are also related to Poincaré inequality, $\log$-Sobolev inequality and hypercontractivity, see $[41,5]$.

Talagrand proved the validity of $T_{2}$ for the multidimensional Gaussian distribution with optimal constant $C=2$. His work was then extended to Wiener measure on the path space in [22]. Transportations inequalities for laws of (forward) SDEs have been extensively studied. We refer to $[15,51,42]$ for equations driven by Brownian motion and to $[47,46]$ for equations driven by fractional Brownian motion or a Gaussian process. All the aforementioned works on SDEs assume that the coefficients are Lipschitz continuous or satisfy a dissipative condition. Note however the exception of [1] who derives versions of the Poincaré and log-Sobolev inequalities of the so-called skew Brownian motion, which can be seen as the solution of an SDE whose drift depends on the local time of the unknown.

Regarding Transportations inequalities for backward SDEs, to the best of the authors' knowledge the only work on the subject is the paper [3] available online since August 2019. It uses the Girsanov transform technique of [15] to derive quadratic transportation 
inequalities for laws of one-dimensional BSDEs with bounded coefficients, and Lipschitz continuous generators.

\subsection{Organization of the paper}

We organize the rest of the paper as follows: The next section is dedicated to the proofs of Theorems 1.1 and 1.3, we also discuss various extensions and applications, including functional inequalities for laws of martingales. In Section 3, we present the proof of Theorem 1.4. The application to portfolio optimization alluded in the introduction is presented in more details. We prove Talagrand inequality for SDEs with measurable drifts in Section 5 and conclude with the analysis of the logarithmic-Sobolev inequality.

\section{The proofs of Theorem 1.3 and Theorem 1.1}

The strategy behind the proofs is to show that the objects of interest are in fact obtained through Lipschitz transformations of Brownian motion. The latter is known to satisfy the $T_{2}$-inequality, see [22, Theorem 3.1]. For this reason, the following lemma on the stability of transportation inequalities under push-forward by Lipschitz maps (taken from [15]) is fundamental and stated separately.

Lemma 2.1 ([15, Lemma 2.1]). Assume that $\mu$ satisfies $T_{2}(C)$ and let $\psi: \Omega \rightarrow \Omega$ be $\mu$-almost surely $L_{\psi}$-Lipschitz. Then the push-forward $\psi_{*} \mu$ satisfies $T_{2}\left(C L_{\psi}^{2}\right)$. by

The proofs need some notational preparation, introduced below. For $t \in[0, T]$ denote

$$
\Omega^{t}:=\left\{\gamma \in C\left([t, T], \mathbb{R}^{d}\right): \gamma(t)=0\right\}
$$

the shifted canonical space, by $W^{t}$ the canonical process on $\Omega^{t}$, by $P^{t}$ the Wiener measure on $\Omega^{t}$, and by $\left(\mathcal{F}_{s}^{t}\right)_{s \in[t, T]}$ the $P^{t}$-completion of the natural filtration of $W^{t}$. For $\omega \in \Omega, t \in[0, T]$, and $\gamma \in \Omega^{t}$ define the concatenation $\omega \otimes_{t} \gamma \in \Omega$ via

$$
\left(\omega \otimes_{t} \gamma\right)(s):= \begin{cases}\omega(s) & \text { if } s \in[0, t), \\ \gamma(s)+\omega(t) & \text { if } s \in[t, T] .\end{cases}
$$

Further, for a function $X: \Omega \times[0, T] \rightarrow \mathbb{R}^{k}$ with $k \in \mathbb{N}$ and fixed $(t, \omega) \in[0, T] \times \Omega$, define its shifts by

$$
X^{t, \omega}: \Omega^{t} \times[t, T] \rightarrow \mathbb{R}^{k}, \quad X_{s}^{t, \omega}(\gamma):=X_{s}\left(\omega \otimes_{t} \gamma\right) .
$$

Similar notation is applied to a function $X: \Omega \rightarrow \mathbb{R}^{k}$ or a function $g: \Omega \times[0, T] \times A \rightarrow \mathbb{R}^{k}$, where $A$ is an arbitrary space, that is, $X^{t, \omega}(\gamma)=X\left(\omega \otimes_{t} \gamma\right)$ or $g_{s}^{t, \omega}(\gamma, a):=g_{s}\left(\omega \otimes_{t} \gamma, a\right)$.

Note that, using the above notation, one has

$$
E\left[X \mid \mathcal{F}_{t}\right](\omega)=\int_{\Omega^{t}} X^{t, \omega}(\gamma) P^{t}(d \gamma)=: E_{P^{t}(d \gamma)}\left[X^{t, \omega}(\gamma)\right]=: E_{P^{t}}\left[X^{t, \omega}\right]
$$

for $P$-almost all $\omega \in \Omega$.

\subsection{Proof of Theorem 1.3 and first consequences}

Proof of Theorem 1.3. It follows from the work of Pardoux \& Peng [44, Theorem 3.1] that the equation (BSDE) admits a unique solution $(Y, Z)$ such that $Z$ is square integrable and $Y$ has ( $P$-almost surely) continuous paths, that is, $Y(\omega) \in C\left([0, T], \mathbb{R}^{m}\right)$ for ( $P$-almost every) $\omega \in \Omega$. Using arguments close in spirit to [19], we will show that the function $\omega \mapsto Y(\omega)$ is Lipschitz continuous.

Let $t \in[0, T]$. By Lemma 2.2 below there is a $P$-zero set $N \subset \Omega$ such that for $\omega \in N^{c}$ one has $Y_{t}(\omega)=Y_{t}^{t, \omega} P^{t}$-almost surely and the pair $\left(Y_{r}^{t, \omega}, Z_{r}^{t, \omega}\right)_{r \in[t, T]}$ satisfies

$$
Y_{r}^{t, \omega}=F^{t, \omega}+\int_{r}^{T} g_{u}^{t, \omega}\left(W^{t}, Y_{u}^{t, \omega}, Z_{u}^{t, \omega}\right) d u-\int_{r}^{T} Z_{u}^{t, \omega} d W_{u}^{t}, \quad P^{t} \text {-a.s. } r \in[t, T] .
$$


From now on fix $\omega, \eta \in N^{c}$ and $t \in[0, T]$. For $r \in[t, T]$ define

$$
\begin{aligned}
\delta Y_{r} & :=Y_{r}^{t, \omega}-Y_{r}^{t, \eta}, \\
\delta Z_{r} & :=Z_{r}^{t, \omega}-Z_{r}^{t, \eta} \\
\delta g_{r} & :=g_{r}^{t, \omega}\left(W^{t}, Y_{r}^{t, \omega}, Z_{r}^{t, \omega}\right)-g_{r}^{t, \eta}\left(W^{t}, Y_{r}^{t, \omega}, Z_{r}^{t, \omega}\right) .
\end{aligned}
$$

As $(y, z) \mapsto g_{r}(\omega, y, z)$ is Lipschitz and therefore Lebesgue almost surely differentiable, it follows that

$$
\begin{aligned}
& g_{r}^{t, \omega}\left(W^{t}, Y_{r}^{t, \omega}, Z_{r}^{t, \omega}\right)-g_{r}^{t, \eta}\left(W^{t}, Y_{r}^{t, \eta}, Z_{r}^{t, \eta}\right) \\
& =\delta g_{r}+\int_{0}^{1}\left(\begin{array}{l}
\partial_{y} g_{r}^{t, \eta}\left(W^{t}, Y_{r}^{t, \omega}-a \delta Y_{r}, Z_{r}^{t, \omega}-a \delta Z_{r}\right) \\
\partial_{z} g_{r}^{t, \eta}\left(W^{t}, Y_{r}^{t, \omega}-a \delta Y_{r}, Z_{r}^{t, \omega}-a \delta Z_{r}\right)
\end{array}\right)^{\top}\left(\begin{array}{l}
\delta Y_{r} \\
\delta Z_{r}
\end{array}\right) d a \\
& :=\delta g_{r}+\beta_{r} \delta Y_{r}+q_{r} \delta Z_{r} .
\end{aligned}
$$

Note that the progressive processes $\beta$ and $q$ are bounded by $L_{g}$. Moreover, the pair $(\delta Y, \delta Z)$ solves the linear equation

$$
\delta Y_{r}=\delta Y_{T}+\int_{r}^{T} \delta g_{u}+\beta_{u} \delta Y_{u}+q_{u} \delta Z_{u} d u-\int_{r}^{T} \delta Z_{u} d W_{u}^{t}, \quad P^{t} \text {-a.s. }
$$

for $r \in[t, T]$, and a standard computation as in [21, Theorem 1.1] reveals that

$$
\delta Y_{r}=e^{\int_{r}^{T} \beta_{u} d u} \delta Y_{T}+\int_{r}^{T} e^{\int_{r}^{u} \beta_{s} d s} \delta g_{u} d u-\int_{r}^{T} e^{\int_{r}^{u} \beta_{s} d s} Z_{u}\left(d W_{u}^{t}-q_{u} d u\right) \quad P^{t} \text { a.s. }
$$

For $r \in[t, T]$, define

$$
\Gamma_{r}:=\exp \left(\int_{t}^{r} q_{u} d W_{u}-\frac{1}{2} \int_{t}^{r}\left|q_{u}\right|^{2} d u\right) \exp \left(\int_{t}^{r} \beta_{u} d u\right) .
$$

By Girsanov's theorem, taking the expectation with respect to the (shifted) measure $P^{t}$ yields

$$
\left|\delta Y_{t}\right|=\left|E_{P^{t}}\left[\Gamma_{T} \delta Y_{T}+\int_{t}^{T} \Gamma_{r} \delta g_{r} d r\right]\right|
$$

$P^{t}$-almost surely. Moreover, by Lipschitz continuity of $g$, it holds that

$$
\begin{aligned}
\left|\delta g_{r}(\gamma)\right| & \leq L_{g}\left\|\omega \otimes_{t} \gamma-\eta \otimes_{t} \gamma\right\|_{\infty} \\
& \leq L_{g}\|\omega-\eta\|_{\infty}
\end{aligned}
$$

for all $\gamma \in \Omega^{t}$. Thus, as $E_{P^{t}}\left[\Gamma_{r}\right] \leq \exp \left(T L_{g}\right)$ for every $r \in[t, T]$, it follows from (2.2) that

$$
\begin{aligned}
\left|\delta Y_{t}\right| & \leq L_{F}\|\omega-\eta\|_{\infty} E_{P^{t}}\left[\Gamma_{T}\right]+L_{g}\|\omega-\eta\|_{\infty} E_{P^{t}}\left[\int_{t}^{T} \Gamma_{r} d r\right] \\
& \leq\left(L_{F}+T L_{g}\right) \exp \left(T L_{g}\right)\|\omega-\eta\|_{\infty}
\end{aligned}
$$

$P^{t}$-almost surely.

As $\omega, \eta \in N^{c}$ and $t \in[0, T]$ were arbitrary and $P(N)=0$, this shows that

$$
Y: \Omega \rightarrow C\left([0, T], \mathbb{R}^{m}\right) \text { is }\left(L_{F}+T L_{g}\right) e^{T L_{g}} \text {-Lipschitz. }
$$

Now recall that by [22, Theorem 3.1], the probability measure $P$ (the law of the Wiener process) satisfies $T_{2}(2)$. Hence, the result follows by Lemma 2.1 . 
Lemma 2.2. For $P$-almost all $\omega \in \Omega$ it holds that

$$
Y_{r}^{t, \omega}=F^{t, \omega}+\int_{r}^{T} g_{u}^{t, \omega}\left(W^{t}, Y_{u}^{t, \omega}, Z_{u}^{t, \omega}\right) d u-\int_{r}^{T} Z_{u}^{t, \omega} d W_{u}^{t}, \quad P^{t} \text {-a.s. } r \in[t, T]
$$

and $Y_{t}(\omega)=Y_{t}^{t, \omega} P^{t}$-almost surely.

Proof. Let $r \geq t$ be fixed and denote by $N_{1}^{c}$ the set of all $\omega \in \Omega$ such that (BSDE) holds true so that $P\left(N_{1}\right)=0$. Then, for every $\omega \in \Omega$ and $\gamma \in \Omega^{t}$ such that $\omega \otimes_{t} \gamma \in N_{1}^{c}$, unwrapping the definitions of $Y^{t, \omega}, Z^{t, \omega}$ and (BSDE) it holds that

$$
Y_{r}^{t, \omega}(\gamma)=F^{t, \omega}(\gamma)+\int_{r}^{T} g_{u}^{t, \omega}\left(\gamma, Y_{u}^{t, \omega}(\gamma), Z_{u}^{t, \omega}(\gamma)\right) d u-\left(\int_{r}^{T} Z_{u} d W_{u}\right)\left(\omega \otimes_{t} \gamma\right)
$$

As the law of the concatenation $\Omega \times \Omega^{t} \ni(\omega, \eta) \mapsto \omega \otimes_{t} \eta \in \Omega$ under $P \otimes P^{t}$ equals $P$, one has

$$
\begin{aligned}
P\left(N_{2}^{c}\right) & =P\left(N_{1}^{c}\right)=1, \text { where } \\
N_{2}^{c} & :=\left\{\omega \in \Omega: P^{t}\left(\gamma \in \Omega^{t}: \omega \otimes_{t} \gamma \in N_{1}^{c}\right)=1\right\} .
\end{aligned}
$$

For every $\omega \in N_{2}^{c}$ we have that (2.3) holds for $P^{t}$-almost all $\gamma \in \Omega^{t}$. Thus we are left to show that for $P$-almost all $\omega \in \Omega$, one has that

$$
\left(\int_{r}^{T} Z_{u} d W_{u}\right)\left(\omega \otimes_{t} \gamma\right)=\left(\int_{r}^{T} Z_{u}^{t, \omega} d W_{u}^{t}\right)(\gamma)
$$

for $P^{t}$-almost all $\gamma \in \Omega^{t}$ and all $r \in[t, T]$.

In case that $Z$ is a simple processes, the $d W$ and $d W^{t}$-integrals are just finite sums. Then, as $r \geq t$, only increments of $\gamma$ appear in either sums and it follows that (2.4) holds true for all $\omega \in \Omega$ and $\gamma \in \Omega^{t}$. In the general case, approximate $Z$ in $L^{2}(P \otimes d u)$ by simple integrands $Z^{n}$ which are progressive w.r.t. the raw filtration (in particular, $Z^{n, t, \omega}$ is progressive w.r.t. $\mathcal{F}^{t}$ and the same holds true for the limit). Using once more that the law of the concatenation under $P \otimes P^{t}$ equals $P$, one obtains that

$$
\begin{aligned}
& E_{P(d \omega)}\left[E_{P^{t}(d \gamma)}\left[\int_{t}^{T}\left|Z_{u}^{n, t, \omega}(\gamma)-Z_{u}^{t, \omega}(\gamma)\right|^{2} d u\right]\right] \\
& =E_{P(d \omega)}\left[\int_{t}^{T}\left|Z_{u}^{n}(\omega)-Z_{u}(\omega)\right|^{2} d u\right] \rightarrow 0 .
\end{aligned}
$$

After passing to a subsequence, one may assume that the inner expectation converges to 0 for $P$-almost all $\omega \in \Omega$, that is $Z^{n, t, \omega} \rightarrow Z^{t, \omega}$ in $L^{2}\left(P^{t} \otimes d u\right)$. The triangle inequality, Ito's isometry, and the fact that the law of $\cdot \otimes_{t} \cdot$ under $P \otimes P^{t}$ equals $P$ then show that

$$
E_{P(d \omega)}\left[E_{P^{t}(d \gamma)}\left[\left|\left(\int_{r}^{T} Z_{u} d W_{u}\right)\left(\omega \otimes_{t} \gamma\right)-\left(\int_{r}^{T} Z_{u}^{t, \omega} d W_{u}^{t}\right)(\gamma)\right|^{2}\right]\right]=0,
$$

which implies (2.4).

To complete the proof, we are left to prove that $Y_{t}(\omega)=Y_{t}^{t, \omega} P^{t}$-almost surely. This is a consequence of Blumenthal's 0-1 law, i.e. conditioning on the right-continuous filtration is up to $P$-zero sets the same as conditioning on the raw filtration. The raw filtration at time $t$ is generated by paths up to time $t$, hence $Y_{t}(\omega)=Y_{t}(\eta)$ for all $\omega, \eta \in \Omega$ such that $\omega=\eta$ on $[0, t]$.

In the next corollary we show that in our Brownian filtration, laws of martingales satisfy transportation inequalities: This corollary as well as the subsequent subsection use the notion of Malliavin derivative. We refer the reader to [39] for an introduction to this topic and the (little bit of) Malliavin calculus used in the article. 
Corollary 2.3. Let $q:[0, T] \times \Omega \rightarrow \mathbb{R}^{d \times m}$ be a bounded progressive process and let $M$ be an $m$-dimensional martingale under the probability measure $Q^{q}:=\operatorname{Law}\left(W+\int q d u\right)$. If $M_{T}$ and $q$ are both Lipschitz continuous in $\Omega$, then the law of $M$ satisfies $T_{2}(C)$ for some constant $C>0$ depending on $d$.

Here ' $q$ is Lipschitz continuous in $\Omega$ ' means that there is a constant $L_{q}$ such that $\left|q_{t}(\omega)-q_{t}(\eta)\right| \leq L_{q}\|\omega-\eta\|_{\infty}$ for all $\omega, \eta \in \Omega$ and $t \in[0, T]$.

Proof. By Lipschitz continuity, $M_{T}$ is square integrable. Thus, it follows from martingale representation and Girsanov's theorem that the process $M$ satisfies

$$
M_{t}=M_{T}+\int_{t}^{T} Z_{u} q_{u} d u-\int_{t}^{T} Z_{u} d W_{u}
$$

for some progressive, square integrable process $Z$. In particular, $(M, Z)$ satisfies the equation (BSDE) with generator $g$ defined by $g_{t}(\omega, y, z):=q_{t}(\omega) z$ and terminal condition $F:=M_{T}$.

As $M_{T}$ is Lipschitz continuous and $q$ of bounded Malliavin derivative (this follows from the Lipschitz assumption on $q$, see e.g. [11, Proposition 3.2]), we have by [11, Theorem 2.2] that $Z$ must be bounded, say by $C$. Thus, $M$ also satisfies (2.5) with the now Lipschitz continuous generator $\tilde{g}_{t}(z):=g_{t}(z) 1_{\{|z| \leq C\}}+g(z C /|z|) 1_{\{|z|>C\}}$. The result then follows from Theorem 1.3.

\subsection{Proof of Theorem 1.1}

We shift our focus on the proof of Theorem 1.1. Denote by $\mathcal{T}^{t}$ the set of all stopping times $\sigma: \Omega^{t} \rightarrow[t, T]$, that is, $\{\sigma \leq s\} \in \mathcal{F}_{s}^{t}$ for all $s \in[t, T]$.

Lemma 2.4. It holds that

$$
\operatorname{ess.sup}_{\tau \text { is stopping time, } t \leq \tau \leq T} E\left[\Gamma_{\tau} \mid \mathcal{F}_{t}\right](\omega)=\sup _{\sigma \in \mathcal{T}^{t}} E_{P^{t}}\left[\Gamma_{\sigma}^{t, \omega}\right]
$$

for $P$-almost all $\omega \in \Omega$.

Proof. In a first step, note that one may restrict everywhere to stopping times w.r.t. the raw filtration. Indeed, for a general stopping time $\tau$ there is a stopping time $\tau^{\prime}$ w.r.t. the right-continuous version of the raw filtration such that $\tau=\tau^{\prime}$ almost surely. Further, integrability and pathwise continuity of $\Gamma$ guarantee that $E\left[\Gamma_{\min \left(\tau^{\prime}+\varepsilon, T\right)}\right] \rightarrow E\left[\Gamma_{\tau^{\prime}}\right]$ as $\varepsilon \rightarrow 0$. It remains to notice that $\min \left(\tau^{\prime}+\varepsilon, T\right)$ is a stopping time w.r.t. the raw filtration for every $\varepsilon>0$. The same arguments apply to conditional expectations.

We start by showing that the left hand side is smaller than the right hand side. To that end, by definition of the essential supremum, there exists a sequence $\left(\tau_{n}\right)$ of stopping times with values in $[t, T]$ such that, $P$-almost surely, the left hand side equals $\sup _{n} E\left[\Gamma_{\tau_{n}} \mid \mathcal{F}_{t}\right]$. For every $n$ and $\omega \in \Omega$ one has that $\tau_{n}^{t, \omega} \in \mathcal{T}^{t}$, which shows that

$$
\begin{aligned}
E\left[\Gamma_{\tau_{n}} \mid \mathcal{F}_{t}\right](\omega) & =E_{P^{t}(d \gamma)}\left[\Gamma_{\tau_{n}^{t, \omega}(\gamma)}^{t, \omega}(\gamma)\right] \\
& \leq \sup _{\sigma \in \mathcal{T}^{t}} E_{P^{t}(d \gamma)}\left[\Gamma_{\sigma(\gamma)}^{t, \omega}(\gamma)\right]
\end{aligned}
$$

for $P$-almost all $\omega$. Taking the countable supremum thus yields the first claim.

As for the reverse inequality, assume first that

$$
\Gamma^{\omega, t}=\sum_{n} f^{n} 1_{A^{n}}(\omega) \quad \text { for every } \omega \in \Omega,
$$


where $\left(A^{n}\right)$ is a $\mathcal{F}_{t}^{0}$-measurable partition of $\Omega$ and $f^{n}$ are functions from $\Omega^{t} \times[t, T]$ to $\mathbb{R}$. Then

$$
\sup _{\sigma \in \mathcal{T}^{t}} E_{P^{t}}\left[\Gamma_{\sigma}^{t, \omega}\right]=\sum_{n} 1_{A^{n}}(\omega) \sup _{\sigma \in \mathcal{T}^{t}} E_{P^{t}}\left[f_{\sigma}^{n}\right]
$$

for every $\omega \in \Omega$. Now, let $\varepsilon>0$ be fixed and, for every $n$, pick some $\sigma^{n} \in \mathcal{T}^{t}$ which achieves the supremum above up to an error of $\varepsilon>0$. Define

$$
\tau: \Omega \rightarrow[t, T] \quad \tau(\omega):=\sum_{n} 1_{A^{n}}(\omega) \sigma^{n}\left(\left.\omega\right|_{[t, T]}-\omega(t)\right),
$$

that is, one has $\tau\left(\omega \otimes_{t} \gamma\right)=\sum_{n} 1_{A^{n}}(\omega) \sigma^{n}(\gamma)$. Then it holds that

$$
E\left[\Gamma_{\tau} \mid \mathcal{F}_{t}\right](\omega)=\sum_{n} 1_{A^{n}}(\omega) E_{P^{t}}\left[f_{\sigma^{n}}^{n}\right] \geq \sup _{\sigma \in \mathcal{T}^{t}} E_{P^{t}}\left[\Gamma_{\sigma}^{\omega, t}\right]-\varepsilon
$$

for $P$-almost all $\omega \in \Omega$. Further, it can be checked that $\tau$ is a stopping time. Hence, under the assumption made on $\Gamma$, the second claim follows.

We are left to argue why this assumption is not restrictive. First, by tightness of $P^{t}$, for every $\varepsilon>0$, there is some compact $K \subset \Omega^{t}$ for which

$$
\left|E_{P^{t}}\left[\Gamma_{\sigma}^{t, \omega}\right]-E_{P^{t}}\left[\Gamma_{\sigma}^{t, \omega} 1_{K}\right]\right| \leq \varepsilon
$$

uniformly over all $\sigma \in \mathcal{T}^{t}$. Now note that $\omega \mapsto \Gamma^{t, \omega} 1_{K}$ is a function with values in the separable space $C(K \times[t, T], \mathbb{R})$. Thus, it can be approximated uniformly by functions of the form $\sum_{n} f^{n} 1_{A^{n}}(\omega)$.

Proof of Theorem 1.1. By [20, Proposition 2.3] one has that $S$ is the value process of the solution of a 'reflected BSDE' with barrier $\Gamma$. Recall that a triple $(Y, Z, K)$ (where $Y$ and $K$ are adapted, $Z$ progressive and $K$ continuous, increasing with $K_{0}=0$ ) solves a reflected BSDE with barrier $\Gamma$ if

$$
\left\{\begin{array}{l}
Y_{t}=F+\int_{t}^{T} g_{s}\left(Y_{s}, Z_{s}\right) d s+K_{T}-K_{t}-\int_{t}^{T} Z_{s} d W_{s} \\
Y_{t} \geq \Gamma_{t} \text { for all } t \in[0, T]
\end{array}\right.
$$

and it holds $\int_{0}^{T}\left(Y_{t}-\Gamma_{t}\right) d K_{t}=0$. Therefore, $S$ has continuous paths.

Let $t \in[0, T]$ be fixed and denote by $N$ the set of all $\omega \in \Omega$ such that

$$
S_{t}(\omega):=\operatorname{ess.sup}_{\tau \in \mathcal{T}_{t}} E\left[\Gamma_{\tau} \mid \mathcal{F}_{t}\right](\omega) \neq \sup _{\sigma \in \mathcal{T}^{t}} E_{P^{t}}\left[\Gamma_{\sigma}^{t, \omega}\right] .
$$

By Lemma 2.4 one has $P(N)=0$. Fix $\omega, \eta \in N^{c}$.

For every $s \in[t, T]$ and $\gamma \in \Omega^{t}$, unwrapping the definition of $X^{t, \omega}$ yields

$$
\begin{aligned}
\left|\Gamma_{s}^{t, \omega}(\gamma)-\Gamma_{s}^{t, \eta}(\gamma)\right| & =\left|\Gamma_{s}\left(\omega \otimes_{t} \gamma\right)-\Gamma_{s}\left(\eta \otimes_{t} \gamma\right)\right| \\
& \leq L_{\Gamma}\left\|\omega \otimes_{t} \gamma-\eta \otimes_{t} \gamma\right\|_{\infty} \\
& \leq L_{\Gamma}\|\omega-\eta\|_{\infty} .
\end{aligned}
$$

Thus, for every $\sigma \in \mathcal{T}^{t}$, it holds that

$$
\left|E_{P^{t}}\left[\Gamma_{\sigma}^{t, \omega}\right]-E_{P^{t}}\left[\Gamma_{\sigma}^{t, \eta}\right]\right| \leq L_{\Gamma}\|\omega-\eta\|_{\infty} .
$$

In particular, as $\omega, \eta \in N^{c}$, this implies that

$$
\left|S_{t}(\omega)-S_{t}(\eta)\right| \leq L_{\Gamma}\|\omega-\eta\|_{\infty} .
$$

Hence, the function $\gamma \mapsto S_{t}(\gamma)$ is $P$-almost surely a $L_{\Gamma}$-Lipschitz function. The claimed functional inequality for (the law of) $S$ follows again from Lemma 2.1 and [22, Theorem 3.1]. 
Corollary 2.5. Let $(Y, Z)$ solve (BSDE) and let $\Gamma:[0, T] \times C\left([0, T], \mathbb{R}^{m}\right) \rightarrow \mathbb{R}$ be an adapted process with continuous paths such that $\Gamma_{t}$ is $L_{\Gamma}$-Lipschitz for every $t \in[0, T]$. Under the conditions of Theorem 1.3 (in case $m \geq 1$ ) or Theorem 1.4 (in case $m=1$ ), the process

$$
S_{t}:=\underset{\tau \text { is stopping time, } t \leq \tau \leq T}{\text { ess.sup }} E\left[\Gamma_{\tau}(Y) \mid \mathcal{F}_{t}\right]
$$

has continuous paths and its law satisfies $T_{2}(C)$ with $C=2 L_{Y}^{2}$ and $L_{Y}=L_{F}+T L_{g} e^{T L_{g}}$ (in case of Theorem 1.3) or $L_{Y}=L_{F}$ (in case of Theorem 1.4).

Proof. Set $\Gamma^{\prime}:=\Gamma \circ Y$. Then $\Gamma^{\prime}$ is still adapted and it has continuous paths. Moreover, from (the proof of) Theorem 1.3 (resp. Theorem 1.4) it follows that $\Gamma_{t}^{\prime}$ is Lipschitz continuous with a constant not depending on $t$. The claim now follows from Theorem 1.1.

\section{The proof of Theorem 1.4}

Recall the definition of the convex conjugate

$$
g_{t}^{*}(q)=\sup _{z \in \mathbb{R}^{d}}\left(q z-g_{t}(z)\right) \text { for every } t \in[0, T] \text { and } q \in \mathbb{R}^{d} .
$$

Remark 3.1. The proof of Theorem 1.4 uses [2, Lemma 5.1], which assumes

$$
\lim _{|q| \rightarrow+\infty} \inf _{t \in[0, T]} \frac{g_{t}^{*}(q)}{|q|}=+\infty \quad \text { and } \quad \int_{0}^{T}\left|\sup _{|q| \leq r} g_{t}^{*}(q)\right| d t<\infty .
$$

When the equation (BSDE) admits a solutions, the conclusion of Theorem 1.4 remains valid if the assumptions (B) and (C) are replaced by the assumptions (3.2). These are in fact weaker assumptions, as the following lemma shows.

Lemma 3.2. Assume that $\sup _{t \in[0, T]} g_{t}(z)<\infty$ for every $z \in \mathbb{R}^{d}$. Then it holds that $\inf _{t \in[0, T]} g_{t}^{*}(q) /|q| \rightarrow \infty$ as $|q| \rightarrow \infty$.

On the other hand, assume that $\inf _{t \in[0, T]} g_{t}(z) /|z| \rightarrow \infty$ as $|z| \rightarrow \infty$. Then it holds that $\sup _{t \in[0, T]} g_{t}^{*}(q)<\infty$ for every $q \in \mathbb{R}^{d}$.

Proof. To show the first claim, let $m \geq 0$ be arbitrary. As $z \mapsto \sup _{t \in[0, T]} g_{t}(z)$ is convex and real valued, it is continuous. Hence, there exists $c>0$ such that $g_{t}(z) \leq c$ for all $|z| \leq m$ and all $t \in[0, T]$. Plugging the choice $z:=m q /|q|$ in (3.1) implies

$$
\inf _{t \in[0, T]} \frac{g_{t}^{*}(q)}{|q|} \geq \inf _{t \in[0, T]}\left(\frac{q z}{|q|}-\frac{g_{t}(z)}{|q|}\right) \geq m-\frac{c}{|q|} \rightarrow m
$$

as $|q| \rightarrow \infty$. Since $m>0$ was arbitrary, this implies the first claim.

To show the second claim, let $q \in \mathbb{R}^{d}$ be arbitrary. We distinguish between small and large $z$ in the representation (3.1). By assumption there is $c>0$ such that $g_{t}(z) \geq 2|q||z|$ for all $t \in[0, T]$ and $|z| \geq c$. For such $z$ one has $q z-g_{t}(z) \leq|z||q|-2|q||z| \leq 0$. On the other hand, $g \geq 0$ implies that $q z-g_{t}(z) \leq c|q|$ for all $|z| \leq c$. This show the second claim.

Proof of Theorem 1.4. In a first step we focus on the case where $F$ is bounded.

It follows from the condition (B) and [31, Theorem 2.3] that equation (BSDE) admits a solution $(Y, Z)$. Let $C_{b}(\Omega)$ denote the space of bounded continuous functions on $\Omega$. For any generator $h$, consider the functional $\rho^{h}: C_{b}(\Omega) \rightarrow \mathbb{R}$ which maps the terminal 
condition $F$ to $Y_{0}$, where $Y$ is the solution of (BSDE) with $g$ substituted by $h$. By [14, Theorems 2.1 and 2.2] and convexity of $g$ one has

$$
Y_{0}=\rho^{g}(F)=\sup _{q} E_{Q}\left[F-\int_{0}^{T} g_{u}^{*}\left(q_{u}\right) d u\right],
$$

where the supremum is taken over all progressive and square integrable processes $q$ with values in $\mathbb{R}^{d}, Q$ a probability measure absolutely continuous with respect to $P$ and with density

$$
\frac{d Q}{d P}:=\exp \left(\int_{0}^{T} q_{u} d W_{u}-\frac{1}{2} \int_{0}^{T}\left|q_{u}\right|^{2} d u\right) .
$$

In particular it follows that

$$
\left|\rho^{g}(F)-\rho^{g}(G)\right| \leq \sup _{\omega \in \Omega}|F(\omega)-G(\omega)|
$$

for all bounded functions $F, G: \Omega \rightarrow \mathbb{R}$.

As in the proofs of Theorems 1.3 and Theorem 1.1 we use shifts of paths, however, this time defined with intrinsic scaling: For $t \in[0, T)$ and $\omega, \eta \in \Omega$ with $\eta(0)=0$, define $\omega \oplus_{t} \eta \in \Omega$ via

$$
\left(\omega \oplus_{t} \eta\right)_{s}:=\omega(t \wedge s)+\sqrt{T-t} \cdot \eta\left(\frac{s-t}{T-t}\right) 1_{[t, T]}(s) .
$$

Only Brownian motion will be plugged in as the second argument, hence $\omega \oplus \eta$ only needs to be defined for paths $\eta$ which start in 0 . However, to be formally correct, one can define $\omega \oplus_{t} \eta:=\omega \oplus_{t}(\eta-\eta(0))$ for all paths $\eta$ which do not start at 0 . Moreover, define $g^{(t)}:[0, T] \times \mathbb{R}^{d} \rightarrow \mathbb{R}$ by

$$
g_{s}^{(t)}(z):=(T-t) g_{t+s(T-t)}\left(\frac{z}{\sqrt{T-t}}\right) .
$$

Since $(Y, Z)$ is the unique solution of (BSDE) with generator $g$, it then follows from [14, Theorem 2.2], [17, Theorem 4.5] and [2, Lemma 5.1] that

$$
Y_{t}(\omega)=\rho^{g^{(t)}}\left(F\left(\omega \oplus_{t} \cdot\right)\right)
$$

for $P$-almost all $\omega \in \Omega$ and every $t \in[0, T)$. Using the 1-Lipschitz continuity of the operator $\rho^{g^{(t)}}(\cdot)$ shown in (3.4) it follows that

$$
\begin{aligned}
\left|Y_{t}(\omega)-Y_{t}(\eta)\right| & \leq \sup _{\gamma \in \Omega}\left|F\left(\omega \oplus_{t} \gamma\right)-F\left(\eta \oplus_{t} \gamma\right)\right| \\
& \leq L_{F} \sup _{\gamma \in \Omega}\left\|\omega \oplus_{t} \gamma-\eta \oplus_{t} \gamma\right\|_{\infty} \\
& \leq L_{F}\|\omega-\eta\|_{\infty}
\end{aligned}
$$

for $P$-almost all $\omega, \eta \in \Omega$ and every $t \in[0, T)$. As $Y_{T}=F$ and $Y$ has $P$-almost surely continuous paths, we conclude that $Y: \Omega \rightarrow \Omega$ is $L_{F}$-Lipschitz $P$-almost surely. It thus follows by [22, Theorem 3.1] and Lemma 2.1 that the law $\mu^{y}$ of $Y$ satisfies $T_{2}\left(2 L_{F}^{2}\right)$.

In case that $F$ is not bounded it follows by Lipschitz continuity of $F$ that it has exponential moments. Denote by $Y^{n}$ the solution to (BSDE) with $F$ replaced by $F \wedge n$ for each $n \in \mathbb{N}$. As $F \wedge n$ is $L_{F}$-Lipschitz, by the above, $Y^{n}: \Omega \rightarrow \Omega$ is $L_{F}$-Lipschitz, and it follows from stability of BSDE with terminal conditions having exponential moments (see [9, Proposition 7]) that $Y^{n} \rightarrow Y P \otimes d t$-almost surely (where $(Y, Z)$ is the solution of (BSDE)). As Lipschitz continuity is stable under pointwise convergence, $Y$ remains $L_{F}$-Lipschitz and the claim again follows from [22, Theorem 3.1] and Lemma 2.1. 
Remark 3.3 (Supersolutions). The condition (B) in Theorem 1.4 serves as a guarantee that the (one-dimensional) BSDE with generator $g$ admits a solution $(Y, Z)$ such that $Y$ satisfies the representation (3.3). Without that condition, the BSDE still admits a unique minimal supersolution $(\bar{Y}, \bar{Z})$ in the sense of [16], and it follows from [17] that $\bar{Y}$ satisfies the representation (3.3). Therefore, the proof of Theorem 1.4 shows that the law of $\bar{Y}$ satisfies $T_{2}\left(C_{y}\right)$ with $C_{y}=2 L_{F}^{2}$.

Corollary 3.4. Assume that

(A) $g:[0, T] \times \mathbb{R}^{d} \rightarrow \mathbb{R}$ is Borel measurable, convex in the last variable, satisfies (B) in Theorem 1.4 and there is $b \in \mathbb{R}$ and bounded Borel $a:[0, T] \rightarrow \mathbb{R}^{d}$ such that $g_{t}(z) \geq a_{t} z+b$.

(B) $F: \Omega \rightarrow \mathbb{R}$ is bounded from below and $L_{F}$-Lipschitz continuous.

Then (BSDE) admits a unique solution $(Y, Z)$ and

$$
\text { the law } \mu^{y} \text { of } Y \text { satisfies } T_{2}\left(C_{y}\right)
$$

with $C_{y}:=2 L_{F}^{2}$.

Proof. Since $(Y, Z)$ satisfies (BSDE), we have

$$
Y_{t}+t b=F+T b+\int_{t}^{T} g_{u}(Z)-\left(a_{u} Z_{u}+b\right) d u-\int_{0}^{T} Z_{u}\left(d W_{u}-a_{u} d u\right) .
$$

By Girsanov's theorem, the process $\tilde{W}:=W-\int_{0}^{t} a_{u} d u$ is a Brownian motion under the probability measure $\tilde{P}$ with density

$$
\frac{d \tilde{P}}{d P}:=\exp \left(-\int_{0}^{T} a_{u} d W_{u}-\frac{1}{2} \int_{0}^{T}\left|a_{u}\right|^{2} d u\right) .
$$

Thus, putting

$$
\tilde{Y}_{t}:=Y_{t}+t b, \quad \tilde{F}:=F+T b \quad \text { and } \quad \tilde{g}_{t}(z):=g_{t}(z)-\left(a_{t} z+b\right),
$$

it holds that $(\tilde{Y}, Z)$ solve equation (BSDE) driven by the Brownian motion $\tilde{W}$ with generator $\tilde{g}$ and terminal condition $\tilde{F}$. In oder words,

$$
\tilde{Y}_{t}=\tilde{F}+\int_{t}^{T} \tilde{g}_{u}\left(Z_{u}\right) d u-\int_{t}^{T} Z_{u} d \tilde{W}_{u} \quad \tilde{P} \text {-a.s. }
$$

Observe that in this case, the function $\tilde{g}$ is convex, positive and satisfies the growth conditions $\tilde{g}_{t}(z) \leq C\left(1+|z|^{2}\right)$ for some $C$ and $\lim _{|q| \rightarrow \infty} \inf _{t \in[0, T]} \tilde{g}(q) /|q|=+\infty$. As argued in the proof of Theorem 1.4, the process $\tilde{Y}$ satisfies $\tilde{Y}_{t}(\omega)=\rho^{\tilde{g}^{(t)}}\left(\tilde{F}\left(\omega \oplus_{t} \cdot\right)\right)$ for a 1Lipschitz continuous operator (depending on $\tilde{P}$ ). In particular, $\omega \mapsto \tilde{Y}(\omega)$ (and therefore $\omega \mapsto Y(\omega))$ is $L_{F}$-Lipschitz continuous and thus, the result follows from [22, Theorem 3.1] and Lemma 2.1.

\subsection{Portfolio optimization}

Let us come back to the quantitative finance application alluded to in Examples 1.7 in the introduction. Consider a market with $m$ stocks whose prices are given by the $m$-dimensional process $S$ following the Black-Scholes model

$$
d S_{t}=S_{t}\left(b_{t} d t+\sigma_{t} d W_{t}\right)
$$


with $b$ and $\sigma$ two bounded functions of $t$ with appropriate dimensions (recall that $W$ is a $d$-dimensional Brownian motion). A basic task in quantitative finance consists of optimizing the expected utility of a given claim by dynamic trading. Concretely, let us fix the exponential utility $U(x):=-\exp (-\alpha x)$ for some $\alpha>0$ modeling the investor's preferences and a claim $F: \Omega \rightarrow \mathbb{R}$. Then the problem in question reads as

$$
u_{t}:=\frac{1}{\alpha} \log \left(-\underset{p}{\operatorname{ess} \sup } E\left[-\exp \left(-\alpha\left(\int_{t}^{T} p_{s}\left(d W_{t}+\theta_{t} d t\right)-F\right)\right) \mid \mathcal{F}_{t}\right]\right) .
$$

Here $\mathcal{A}$ is the set of admissible strategies, i.e. the set of all predictable processes $p$ with values in a convex and closed set $\mathbb{A} \subseteq \mathbb{R}^{d}$ for which $E\left[\int_{0}^{T}\left|p_{t}\right|^{2} d t\right]<\infty$ and $\left\{\exp \left(-\alpha \int_{0}^{\tau} p_{t}\left(d W_{t}+\theta_{t} d t\right)\right): \tau\right.$ is stopping time $\}$ is uniformly integrable, and $\theta_{t}:=$ $\sigma^{t r}\left(\sigma_{t} \sigma_{t}^{t r}\right)^{-1} b_{t}$. In particular, $\sigma_{t} \sigma_{t}^{t r}$ is invertible and we assume moreover that $\theta$ is a bounded function of time only.

Theorem 3.5 ([29, Theorem 7 and Proposition 9]). Assume that $F$ is bounded. There is an admissible portfolio $p^{*}$ which is optimal for all $t$ simultaneously (i.e. $p^{*}$ achieves (3.5) for every $t \in[0, T])$.

Moreover, defining $g$ by $g_{t}(\omega, y, z)=(\alpha / 2) \operatorname{dist}^{2}\left(z+\theta_{t} / \alpha, \mathbb{A}\right)-z \theta_{t}-\left|\theta_{t}\right|^{2} /(2 \alpha)$, the pair $\left(u, p^{*}-\theta / \alpha\right)$ solves (BSDE) with generator $g$ and terminal condition $F$.

Since the constraint set $\mathbb{A}$ is convex, the generator $g$ satisfies the conditions of Corollary 3.4. Thus, if $F$ is Lipschitz continuous, then the law of $u$ satisfies $T_{2}(C)$ for some constant $C>0$. If $\mathbb{A}=\mathbb{R}^{d}$ and we additionally assume that $F$ is Malliavin differentiable with Lipschitz continuous Malliavin differentials, then by Corollary 4.5 below the law of $p^{*}$ satisfies $T_{2}$ as well.

These imply that (under the above assumptions made on $F$ and $\mathbb{A}$ ), convergence of empirical measure as in Corollary 1.2 can be deduced, or the Gaussian concentration

$$
\begin{gathered}
P\left(\left|f\left(u_{t}\right)-E\left[f\left(u_{t}\right)\right]\right| \geq x\right) \leq 2 \exp \left(-c x^{2} / L_{f}^{2}\right), \\
P\left(\left|g\left(p_{t}^{*}\right)-E\left[g\left(p_{t}^{*}\right)\right]\right| \geq x\right) \leq 2 \exp \left(-c x^{2} / L_{g}^{2}\right)
\end{gathered}
$$

for every $x>0$ and $L_{f}$-Lipschitz continuous functions $f$ and $g$ of appropriate dimensions.

\section{Transportation inequalities for the control process}

This section presents (modest) results on transportation inequalities for the control process $Z$. Its main finding is Corollary 4.5 which shows that for a linear equation the law of $Z$ satisfies the $T_{2}$ inequality.

We use the notation $D_{s}^{i} \xi$ for the Malliavin derivative of the random variable $\xi$ in the direction of the $i^{\text {th }}$ Brownian motion.

Lemma 4.1. In addition to the assumptions of Theorem 1.3, assume that

(C) We have $g .(0,0,0) \in L^{2}([0, T])$, for every $(t, y, z)$, the function $\omega \mapsto g_{t}(\omega, y, z)$ is Malliavin differentiable and it holds

$$
\left|D_{u}^{i} g_{t}\left(\omega, y^{1}, z^{1}\right)-D_{u}^{i} g_{t}\left(\omega, y^{2}, z^{2}\right)\right| \leq K_{u}(t)\left(\left|y^{1}-y^{2}\right|+\left|z^{1}-z^{2}\right|\right)
$$

for every $\omega \in \Omega, y^{1}, y^{2} \in \mathbb{R}^{m}, z^{1}, z^{2} \in \mathbb{R}^{m \times d}, t, u \in[0, T]$ and $i=1, \ldots, d$ and for some $\mathbb{R}_{+}$-valued adapted process $\left(K_{u}(t)\right)_{t, u \in[0, T]}$ such that we have $\int_{0}^{T} E\left[\left(\int_{0}^{T}\left|K_{u}(t)\right|^{2} d t\right)^{2}\right] d u<\infty$.

(D) The function $F$ is Malliavin differentiable and $t \mapsto D_{t}^{i} F$ is continuous. 
Functional inequalities for forward and backward diffusions

Then $Z$ has continuous paths and is bounded. In particular, the law $\mu^{z}$ of $Z$ satisfies

$$
\mathcal{W}_{2}\left(\mu^{z}, \nu\right) \leq C_{z} H\left(\nu \mid \mu^{z}\right)^{1 / 4} \text { for all } \nu \in \mathcal{P}(\Omega),
$$

with $C_{z}:=2\left(1+\left(m L_{F}^{2} e^{\left(L_{g}+1\right)^{2} T}+m L_{g}^{2} T\right)^{4}\right)^{1 / 4}$.

Remark 4.2. Notice that the Malliavin differentiability of $g$ and $F$ are consequences of the Lipschitz continuity assumptions made in Theorem 1.3. The additional property needed in Lemma 4.1 is the regularity of $D_{u}^{i} g$.

Proof. First assume that the function $g$ is continuously differentiable in $(y, z)$. Since $g$ is $L_{g}$-Lipschitz continuous, it follows by [11, Proposition 3.2] that $g_{t}(\cdot, y, z)$ is Malliavin differentiable for every $t, y, z$ and $\left|D_{s}^{i} g_{t}(y, z)\right| \leq L_{g}$ for all $i=1, \ldots, d$. Similarly, $F$ is Malliavin differentiable and for every $t \in[0, T]$, it holds that $\left|D_{t}^{i} F\right| \leq L_{F}$ for all $i=1, \ldots, d$. Thus, since $t \mapsto D_{t}^{i} F$ is continuous, it follows by [21, Proposition 5.3], that the process $Z$ has a version with continuous paths. Moreover, $Z$ is bounded, see [33, Lemma 3.2]. In fact, it is shown therein that $Z$ satisfies

$$
\left|Z_{t}\right|^{2} \leq m L_{F}^{2} e^{\left(2 L_{g}+L_{g}^{2}+1\right) T}+m L_{g}^{2} T=: C .
$$

If $g$ is not continuously differentiable in $(y, z)$, let $g^{n}$ be a sequence of smooth functions converging to $g$ and denote by $\left(Y^{n}, Z^{n}\right)$ the solution of equation (BSDE) with generator $g^{n}$. Then, it follows for instance by [21, Proposition 2.1] that $Z^{n}$ converges to $Z$ in $L^{2}(P \otimes d t)$. Therefore, $Z$ also satisfies (4.2).

In particular, it has exponential moments of all orders. Thus, it follows by [6, Corollary 2.4] that the law $\mu^{z}$ of $Z$ satisfies (4.1).

In the next corollary, we show that when the Malliavin derivative of the function $g$ is bounded, it does not need to be Lipschitz continuous in $z$ in order to have a transportation inequality for the law of $Y$. In fact, the function $g$ can grow arbitrarily fast in its last variable.

Corollary 4.3. Assume that $g:[0, T] \times \Omega \times \mathbb{R}^{m} \times \mathbb{R}^{m \times d} \rightarrow \mathbb{R}^{m}$ satisfies

(A) There is an increasing function $\varphi: \mathbb{R}_{+} \rightarrow \mathbb{R}_{+}$such that for every $\omega^{1}, \omega^{2} \in \mathcal{C}$, $y^{1}, y^{2} \in \mathbb{R}^{m}, z^{1}, z^{2} \in \mathbb{R}^{m \times d}$ it holds

$$
\left|g_{t}\left(\omega^{1}, y^{1}, z^{1}\right)-g_{t}\left(\omega^{2}, y^{2}, z^{2}\right)\right| \leq L_{g}\left(|| \omega^{1}-\omega^{2} \|_{\infty}+\left|y^{1}-y^{2}\right|\right)+\varphi\left(\left|z^{1}\right| \vee\left|z^{2}\right|\right)\left|z^{1}-z^{2}\right| .
$$

(B) $F: \Omega \rightarrow \mathbb{R}^{m}$ is $L_{F}$-Lipschitz continuous.

(C) Condition (C) in Lemma 4.1 is satisfied.

Then, if $T$ is small enough the equation (BSDE) admits a unique solution $(Y, Z)$ and

$$
\text { the law } \mu^{y} \text { of } Y \text { satisfies } T_{2}\left(C_{y}\right)
$$

with $C_{y}:=2\left(L_{F}+T \max \left(L_{g}, \rho(Q)\right)\right)^{2} e^{2 T \max \left(L_{g}, \varphi(\Lambda)\right)}$, where $\Lambda$ is given by $\Lambda:=\left(2 d m\left(L_{F}^{2}+\right.\right.$ $\left.\left.T L_{g}^{2}\right)\right)^{1 / 2}$.

Furthermore, if the map $t \mapsto D_{t}^{i} F$ is continuous, then the law $\mu^{z}$ of $Z$ satisfies

$$
\mathcal{W}_{2}\left(\mu^{z}, \nu\right) \leq C_{z} H\left(\nu \mid \mu^{z}\right)^{1 / 4} \text { for all } \nu \in \mathcal{P}(\Omega),
$$

with $C_{z}:=2\left(1+\left(m L_{F}^{2} e^{\left(\max \left(L_{g}, \varphi(\Lambda)\right)+1\right)^{2} T}+m T \max \left(L_{g}, \varphi(\Lambda)\right)^{2}\right)^{4}\right)^{1 / 4}$. 
Proof. As argued in the proof of Lemma 4.1, $F$ is Malliavin differentiable and has a derivative bounded by $L_{F}$. Therefore, the existence of a unique solution $(Y, Z)$ follows from [33, Theorem 3.1], where it is further proved that the process $Z$ satisfies $|Z| \leq \Lambda$, provided that $T \leq \frac{\log (2)}{2 L_{g}+\varphi^{2}(\Lambda)+1}$. The truncated function

$$
\hat{g}_{t}(y, z):=\left\{\begin{array}{l}
g_{t}(y, z) \text { if }|z| \leq \Lambda \\
g_{t}(y, \Lambda z /|z|) \text { if }|z|>\Lambda
\end{array}\right.
$$

is Lipschitz continuous with Lipschitz constant smaller than $\max \left(L_{g}, \varphi(\Lambda)\right)$, and since $g_{t}\left(Y_{t}, Z_{t}\right)=\hat{g}_{t}\left(Y_{t}, Z_{t}\right) P$-almost surely for every $t \in[0, T]$ we conclude by uniqueness that $(Y, Z)$ solves the equation (BSDE) with $g$ replaced by $\hat{g}$. Thus, the results follow by Theorem 1.3 and Lemma 4.1 .

Remark 4.4. When the function $g$ is deterministic, it is automatically Malliavin differentiable and its Malliavin derivative is zero. In this case, all the conditions pertaining to the Malliavin derivative of $g$ in Lemma 4.1 and Corollary 4.3 are trivially satisfied.

On the other hand, the smallness condition on the time horizon $T$ is necessary because $Y$ is a multidimensional process. It is well known that in the multidimensional case, and when the function $g$ is allowed to grow as fast as the quadratic function, backward SDE are typically ill-posed for arbitrary time horizons, see for instance [23] for a discussion of this issue. The smallness condition is not necessary in one dimension.

In Lemma 4.1 and Corollary 4.3 we derived a transportation inequality of the form $W_{2}\left(\mu^{z}, \nu\right) \leq \phi\left(H\left(\mu^{z} \mid \nu\right)\right)$ for the law of $Z$, with $\phi(x)=x^{1 / 4}$. While this type of inequality (extensively studies e.g. in [27]) allow to derive deviation inequalities, they do not tensorize or allow to derive other important inequalities as Poincaré inequality.

The next corollary provides a simple example under which Talagrand inequality holds for the law of $Z$. It is the case of a linear equation.

Corollary 4.5. Assume that

(A) $F: \Omega \rightarrow \mathbb{R}^{m}$ is Malliavin differentiable and its Malliavin derivatives $D^{i} F_{t}: \Omega \rightarrow \mathbb{R}^{m}$ are $L_{F}$-Lipschitz continuous.

(B) $g_{t}(\omega, y, z)=\alpha_{t}(\omega)+\beta y+\gamma z$ for some constants $\beta, \gamma$ and a progressive, square integrable process $\alpha$ such that for each $t, \alpha_{t}$ is Malliavin differentiable and its derivative are $L_{\alpha}$-Lipschitz continuous.

Let $(Y, Z)$ be the unique solution of equation (BSDE). Then, the

$$
\text { the law } \mu^{z} \text { of } Z \text { satisfies } T_{2}\left(C_{z}\right)
$$

with $C_{z}:=2\left(L_{F}+T L_{G}\right)^{2} e^{2 T L_{G}}$ and $L_{G}:=\max \left(L_{\alpha},|\beta|,|\gamma|\right)$.

If in addition $F$ and $\alpha_{t}$ are respectively $L_{F}$ - and $L_{\alpha}$-Lipschitz continuous, then

$$
\text { the law } \mu^{y, z} \text { of }(Y, Z) \text { satisfies } T_{2}\left(C_{y, z}\right)
$$

with $C_{y, z}:=\max \left(C_{y}, C_{z}\right)$.

Proof. It follows from [21] that for every $t \in[0, T]$, the pair $\left(Y_{t}, Z_{t}\right)$ is Malliavin differentiable and a version of the derivatives $\left(D_{s}^{i} Y_{t}, D_{s}^{i} Z_{t}\right), i=1, \ldots, d$ satisfies the linear equations

$$
D_{s}^{i} Y_{t}=D_{s}^{i} F+\int_{t}^{T} D_{s}^{i} \alpha_{r}+\beta D_{s}^{i} Y_{r}+\gamma D_{s}^{i} Z_{r} d r-\int_{t}^{T} D_{s}^{i} Z_{r} d W_{r}
$$


for $i=1, \ldots, d$ and $D_{t}^{i} Y_{t}=Z_{t}^{i}$. Moreover, by (B), the function $G(\omega, y, z)$ given by

$$
G(\omega, y, z):=D_{s}^{i} \alpha_{r}(\omega)+\beta y+\gamma z
$$

is Lipschitz continuous, with Lipschitz constant $L_{G}:=\max \left(L_{\alpha},|\beta|,|\gamma|\right)$. Thus, it follows by Theorem 1.3 (and its proof) that the process $\left(D_{t} Y_{t}\right)_{t}$ is a Lipschitz continuous function of $W$, and its law satisfies $T_{2}\left(C_{z}\right)$. Since $Z_{t}=D_{t} Y_{t} P \otimes d t$-almost surely, the first claim follows.

If $\alpha$ and $F$ are Lipschitz continuous, then it follows by Theorem 1.3 that $Y=\phi(\omega)$ for some Lipschitz continuous function $\phi$. Thus, the second claim follows by Lemma 2.1 since $(Y, Z)$ is a Lipschitz continuous function of $W$.

\section{Proof for SDEs}

Proof of Theorem 1.8. It follows from the conditions (B) and (C) that the function $b$ is bounded. Thus, the existence of a unique strong solution follows from [54, Theorem 4].

Define the following three functions

$$
\begin{aligned}
f_{t}(x) & :=\exp \left(\int_{-\infty}^{x}-2 \frac{b_{t}(a)}{\sigma_{t} \sigma_{t}^{\prime}(a)} d a\right), \\
F_{t}(x) & :=\int_{0}^{x} f_{t}(a) d a, \text { and } \\
G_{t}(x) & :=\left[F_{t}(\cdot)\right]^{-1}(x)
\end{aligned}
$$

for all $(t, x) \in[0, T] \times \mathbb{R}$. Note that it follows from the integrability assumption (C) that $F_{t}(\cdot)$ is bijective and therefore $G$ is well-defined. Moreover, as $b .(x)$ and $\sigma .(x)$ are differentiable by assumption (A) and (B), it follows from (C) that $f .(x)$ (and therefore $F .(x)$ ) is differentiable for every $x \in \mathbb{R}$. Further $F_{t}(\cdot)$ is differentiable with derivative $\partial_{x} F_{t}(x)=f_{t}(x)$ for every $(t, x) \in[0, T] \times \mathbb{R}$ and $\partial_{x} F_{t}(\cdot)$ is absolutely continuous w.r.t. Lebesgue measure for every $t \in[0, T]$. Thus, it admits a weak derivative which we denote $\partial_{x x} F_{t}(\cdot)$. That is, $F$ belongs to the Sobolev space $W_{2}^{1,2}([0, T] \times \mathbb{R})$.

Putting $Y_{t}:=F_{t}\left(X_{t}\right)$, it follows from Itô-Krylov's formula [32, Theorem 2.10.1] that

$$
\begin{aligned}
Y_{t} & =Y_{0}+\int_{0}^{t} \partial_{t} F_{s}\left(X_{s}\right)+\frac{1}{2} \partial_{x x} F_{s}\left(X_{s}\right) \sigma_{s} \sigma_{s}^{\prime}\left(X_{s}\right) d s+\int_{0}^{t} \partial_{x} F_{s}\left(X_{s}\right) b_{s}\left(X_{s}\right) d W_{s} \\
& =Y_{0}+\int_{0}^{t} \partial_{t} F_{s}\left(G_{s}\left(Y_{s}\right)\right) d s+\int_{0}^{t} f_{s}\left(G_{s}\left(Y_{s}\right)\right) \sigma_{s}\left(G_{s}\left(Y_{s}\right)\right) d W_{s} .
\end{aligned}
$$

By assumption (C) one has $\exp \left(-c_{1}\right) \leq f \leq \exp \left(c_{1}\right)$. Hence, for every $t \in[0, T]$, both mappings

$$
F_{t}(\cdot) \quad \text { and } \quad G_{t}(\cdot) \text { are } \exp \left(c_{1}\right) \text {-Lipschitz. }
$$

Further, as $\left|\partial_{t} f\right|$ is bounded by $\exp \left(c_{1}\right) c_{3}$, it follows that

$$
\partial_{t} F_{t}(\cdot)=\int_{0}^{\cdot} \partial_{t} f_{t}(a) d a \text { is } c_{3} \exp \left(c_{1}\right) \text {-Lipschitz }
$$

for every $t \in[0, T]$. Thus $\partial_{t} F_{t}(G(t, \cdot))$ is $c_{3} \exp \left(2 c_{1}\right)$-Lipschitz for every $t \in[0, T]$. Moreover

$f_{t}(\cdot)$ is $c_{2} \exp \left(c_{1}\right)$-Lipschitz,

$f_{t}\left(G_{t}(\cdot)\right)$ is $c_{2} \exp \left(2 c_{1}\right)$-Lipschitz, and

$\sigma_{t}\left(G_{t}(\cdot)\right)$ is $L_{\sigma} \exp \left(c_{1}\right)$-Lipschitz 
for every $t \in[0, T]$. As $f$ is bounded by $\exp \left(c_{1}\right)$ and $\sigma$ by $\|\sigma\|_{\infty}$, one obtains that the product

$$
f_{t}\left(G_{t}(\cdot)\right) \sigma_{t}\left(G_{t}(\cdot)\right) \text { is }\|\sigma\|_{\infty} c_{2} \exp \left(2 c_{1}\right)+\exp \left(2 c_{1}\right) L_{\sigma} \text {-Lipschitz }
$$

for every $t \in[0, T]$.

Therefore, [51, Theorem 1] shows that the law $\mu^{y}$ of $Y$ satisfies $T_{2}\left(C_{y}\right)$ with

$$
C_{y}=6 \exp \left(15 \max \left(c_{3} \exp \left(2 c_{1},\|\sigma\|_{\infty} c_{2} \exp \left(2 c_{1}\right)+\exp \left(2 c_{1}\right) L_{\sigma}^{2}\right)\right)\right) .
$$

Moreover, by the above, $X=G(Y)$ and the mapping $G$ is $\exp \left(c_{1}\right)$-Lipschitz. An application of Lemma 2.1 shows that $\mu^{x}$ satisfies $T_{2}\left(\exp \left(c_{1}\right) C_{y}\right)$.

Example 5.1. Let us come back to the Langenvin dynamic presented in the introduction, see (1.2). These equations play a fundamental role notably in the modeling of physical phenomena, see for instance the survey of [48] for applications in Physics, and the more recent mathematical treatments [30,13]. It follows by Theorem 1.8 that if $U^{\prime}$ is integrable and bounded, then the law of $X$ satisfies $T_{2}(C)$ for some $C>0$.

It is well-known that if $U$ grows fast enough for $e^{-\lambda U}$ to be integrable, (and it holds $x U^{\prime}(x) \geq c_{1} x^{2}-c_{2}$ for some $c_{1}, c_{2} \geq 0$ ) then the measure $\mu$ on $\mathbb{R}$ with density (with respect to Lebesgue measure) $e^{-\lambda U(x)} / \int_{\mathbb{R}} e^{-\lambda U(x)} d x$ is the unique stationary measure of the solution of the Langenvin equation, see e.g. [35, Lemma 1.2]. Since $U^{\prime}$ does not depend on time, the constant $C_{x}$ in Theorem 1.8 is time independent. Therefore, it follows by Theorem 1.8 that the measure $\mu$ satisfies $T_{2}(C)$.

It is well-known, see e.g. [36, Theorem 5.2], that log-concave measures ${ }^{1}$ satisfy the quadratic transportation inequality. Notice that in the above arguments we do not require $U$ to be convex, but simply a differentiable function on the real line. Nevertheless, a standard tensorization argument for $T_{2}$ inequalities allows to extend the argument to measures on $\mathbb{R}^{d}$ with density of the form $e^{-\sum_{i=1}^{d} U\left(x_{i}\right)}$.

\section{Logarithmic-Sobolev inequality}

In this final section we prove the logarithmic-Sobolev inequality for the law of $Y_{t}$. This requires some notational preparations.

Let $H$ denote the Cameron-Martin space

$$
H:=\left\{h \in \Omega: h \text { is absolutely continuous, } h_{0}=0 \text { and } \int_{0}^{T}\left|\dot{h}_{s}\right|^{2} d s<\infty\right\} .
$$

Then $H$ becomes a Hilbert space when equipped with the inner product $\langle h, g\rangle_{H}:=$ $\int_{0}^{T} \dot{h}_{s} \dot{g}_{s} d s$ for $h, g \in H$ and associated norm $\|h\|_{H}:=\langle h, h\rangle_{H}^{1 / 2}$. Let $F: \Omega \rightarrow \mathbb{R}$ be Malliavin differentiable, with $D_{t} F \in L^{2}$ for all $t \in[0, T]$. Given $h \in H$, consider the directional derivative

$$
D^{h} F(\omega):=\lim _{\varepsilon \downarrow 0} \frac{F(\omega+\varepsilon h)-F(\omega)}{\varepsilon} .
$$

This defines a continuous linear operator on $H$, so that by Riesz representation theorem, there is a map $\omega \mapsto \bar{D}_{s} F(\omega)$ with values in $H$ such that

$$
D^{h} F(\omega)=\langle\bar{D} F(\omega), h\rangle_{H} .
$$

It is well-known that $\bar{D} F=D F P$-almost surely, see e.g. [52, Remark B.6.2]. In particular, as $H$ is separable, this implies that $|D F|_{H}=\sup _{h \in H \text { s.th. }|h|_{H} \leq 1}\left|D^{h} F\right| P$-almost surely.

\footnotetext{
${ }^{1} \mathrm{~A}$ probability measure $\mu$ is said to be log-concave if its density with respect to Lebesgue measure is of the form $e^{-U}$ with $U$ convex.
} 
The following Lemma is in spirit the same as Lemma 2.1. It states that, since the Wiener measure $P$ satisfies the log-Sobolev inequality

$$
\operatorname{Ent}_{P}(f):=\int f^{2} \log \left(\frac{f^{2}}{\int f^{2} d P}\right) d P \leq 2 \int_{\Omega}|D f|_{H}^{2} d P
$$

for every $P$-integrable and Malliavin differentiable function $f: \Omega \rightarrow \mathbb{R}^{m}$, the push forward of the Wiener measure under any Lipschitz transformation to the Euclidean space again satisfies the log-Sobolev inequality. It is likely to be known, but we could not find a reference and therefore provide its proof. Note however that the finite dimensional case is given in [12, Section 1].

Lemma 6.1. Let $\psi:\left(\Omega,\|\cdot\|_{\infty}\right) \rightarrow \mathbb{R}^{m}$ be $L_{\psi}$-Lipschitz continuous. Then $\psi_{*} P$ satisfies $\operatorname{LSI}\left(2 T L_{\psi}^{2}\right)$.

Proof. In a first step, note that by Lipschitz continuity of $\psi$, it is Malliavin differentiable (with derivative bounded by $L_{\psi}$ ), see e.g. [11, Proposition 3.2]. Let $f: \mathbb{R}^{m} \rightarrow \mathbb{R}$ be differentiable. We need to show that $\operatorname{Ent}_{\psi_{*} P}(f) \leq 2 T L_{\psi}^{2} \int|\nabla f| d\left(\psi_{*} P\right)$.

To that end, let $\omega \in \Omega$ and $h \in H$ be arbitrary and note that

$$
\begin{aligned}
& \limsup _{\varepsilon \downarrow 0}\left|\frac{f(\psi(\omega+\varepsilon h))-f(\psi(\omega))}{\varepsilon}\right| \\
& \leq \limsup _{\varepsilon \downarrow 0}\left|\nabla f(\psi(\omega)) \cdot \frac{\psi(\omega+\varepsilon h)-\psi(\omega)}{\varepsilon}\right| \leq|\nabla f(\psi(\omega))| \cdot L_{\psi}\|h\|_{\infty}
\end{aligned}
$$

by $L_{\psi}$-Lipschitz continuity of $\psi$ and the Cauchy-Schwarz inequality. Further, Hölder's inequality implies that $\|h\|_{\infty} \leq \sqrt{T}|h|_{H}$, hence

$$
D^{h}(f \circ \psi)(\omega) \leq L_{\psi} \sqrt{T} \cdot|\nabla f(\psi(\omega))| \cdot|h|_{H}
$$

for every $h \in H$. By the discussion preceding the lemma, this therefore implies that

$$
|D(f \circ \psi)|_{H} \leq L_{\psi} \sqrt{T} \cdot|\nabla f \circ \psi|
$$

$P$-almost all surely.

Now notice that $\operatorname{Ent}_{P}(f \circ \psi)=\operatorname{Ent}_{\psi_{*} P}(f)$ by the transformation lemma. Hence, as the Wiener meausre $P$ satisfies $L S I(2)$ by [28] (see also [10] and [25, Theorem 1.1] for a formulation using the Malliavin gradient as ours), it follows that

$$
\begin{aligned}
\operatorname{Ent}_{\psi_{*} P}(f) & =\operatorname{Ent}_{P}(f \circ \psi) \\
& \leq 2 \int_{\Omega}|D(f \circ \psi)|_{H}^{2} d P \leq 2 T L_{\psi}^{2} \int_{\mathbb{R}^{m}}|\nabla f| d\left(\psi_{*} P\right) .
\end{aligned}
$$

This proves the claim.

Proof of Theorem 1.11. Recall from the proof of Theorem 1.3 that $Y: \Omega \rightarrow \Omega$ is $L_{Y^{-}}$ Lipschitz with $L_{Y}=\sqrt{C_{y} / 2}$ (where $C_{y}$ is the constant given in that theorem). In particular $Y_{t}: \Omega \rightarrow \mathbb{R}^{m}$ remains $L_{Y}$-Lipschitz. The proof is completed by an application of Lemma 6.1 .

\section{References}

[1] A. T. Abakirova. On some functional inequalities for skew Brownian motion. Proceedings of the Stekov Institute of Mathematics, 287:3-13, 2014. MR-3484320

[2] J. Backhoff-Veraguas, D. Lacker, and L. Tangpi. Non-exponential Sanov and Schilder theorems on Wiener space: BSDEs, Schrödinger problems and control. Ann. Appl. Probab., to appear, 2018. 
[3] K. Bahlali, B. Boufoussi, and S. Mouchtabih. Transportation cost inequality for backward stochastic differential equations. Stati. Probab. Lett., 155:108586, 2019. MR-3994484

[4] S. Becker, P. Cheridito, and A. Jentzen. Deep optimal stopping. Journal of Machine Learning Research, 20(74):1-25, 2019. MR-3960928

[5] S. Bobkov, I. Gentil, and M. Ledoux. Hypercontractivity of Hamilton-jacobi equations. J. Math. Pure Appl., 9(80):669-696, 2001. MR-1846020

[6] F. Bolley and C. Villani. Weighted Csiszár-Kllback-Pinsker inequalities and applications to transportation inequalities. Ann.Fac. Sci. Toulouse Math., XIV(3):331-352, 2005. MR-2172583

[7] S. Boucheron, G. Lugosi, and P. Massart. Concentration Inequalities: A Nonasymptotic Theory of Independence. Oxford University Press, 2013. MR-3185193

[8] S. Boucheron and M. Thomas. Concentration inequalities for order statistics. Electr. Comm. Probab., 17(51):1-12, 2012. MR-2994876

[9] P. Briand and Y. Hu. Quadratic BSDEs with convex generators and unbounded terminal conditions. Probab. Theory Related Fields, 141(3-4):543-567, 2008. MR-2391164

[10] M. Capitaine, E. P. Hsu, and M. Ledoux. Martingale representation and a simple proof of Logarithmic-Sobolev inequalities on path spaces. Electr. Comm. Probab., 2:71-81, 1997. MR-1484557

[11] P. Cheridito and K. Nam. BSDEs with terminal conditions that have bounded Malliavin derivative. J. Funct. Anal., 266(3):1257-1285, 2014. MR-3146818

[12] M. Colombo, A. Figalli, and Y. Jhaveri. Lipschitz changes of variables between perturbations of log-concave measures. Ann. Sc. Norm. Super. Pisa Cl. Sci., 17:1491-1519, 2017. MR-3752535

[13] G. Conforti and M. Von Renesse. Couplings, gradient estimates and logarithmic Sobolev inequalitiy for langevin bridges. Probab. Theory Related Fields, 172(1):493-524, 2018. MR3851837

[14] F. Delbaen, Y. Hu, and X. Bao. Backward SDEs with Superquadratic Growth. Probab. Theory Relat. Field, 150(1-2):145-192, 2011. MR-2800907

[15] H. Djellout, A. Guillin, and L. Wu. Transportation cost-information inequalities and applications to random dynamical systems and diffusions. Ann. Probab., 32(3B):2702-2732, 2004. MR-2078555

[16] S. Drapeau, G. Heyne, and M. Kupper. Minimal supersolutions of convex BSDEs. Ann. Probab., 41(6):3697-4427, 2013. MR-3161467

[17] S. Drapeau, M. Kupper, E. Rosazza Gianin, and L. Tangpi. Dual representation of minimal supersolutions of convex BSDEs. Ann. Inst. H. Poincaré Probab. Statist., 52(2):868-887, 2016. MR-3498013

[18] D. P. Dubhashi and A. Panconesi. Concentration of Measure for the Analysis of Random Algorithms. Cambridge University Press, 2012. MR-2547432

[19] I. Ekren, C. Keller, N. Touzi, and J. Zhang. On viscosity solutions of path depended PDEs. Ann. Probab, 42(1):204-236, 2014. MR-3161485

[20] N. El Karoui, C. Kapoudjian, E. Pardoux, S. Peng, and M. Quenez. Reflected solutions of backward SDE's, and related obstacle problems for PDE's. Ann. Probab., 25(2):702-737, 1999. MR-1434123

[21] N. El Karoui, S. Peng, and M. C. Quenez. Backward stochastic differential equations in finance. Math. Finance, 1(1):1-71, 1997. MR-1434407

[22] D. Feyel and A. S. Üstünel. Monge-Kantorovitch measure transportation and Monge-Ampère equation on Wiener space. Probab. Theory Relat. Fields, 128(3):347-385, 2004. MR-2036490

[23] C. Frei and G. Dos Reis. A financial market with interacting investors: does an equilibrium exist? Math. Financ. Econ., 4(3):161-182, 2011. MR-2796281

[24] M. Fuhrman and G. Tessitore. Nonlinear Kolmogorov equations in infinite dimension spaces: The backward stochastic differential equations approach and applications to optimal control. Ann. Probab., 30(3):1397-1465, 2002. MR-1920272

[25] M. Gourcy and L. Wu. Logarithmic Sobolev inequalities of diffusions for $l^{2}$ metric. Potential Anal., 25(1):77-102, 2006. MR-2238937 
[26] N. Gozlan. A characterization of dimension free concentration in terms of transporation inequalities. Ann. Probab., 37(6):2480-2498, 2009. MR-2573565

[27] N. Gozlan and C. Léonard. A large deviation approach to some transportation cost inequalities. Probab. Theory Relat. Fields, 139(1-2):235-283, 2007. MR-2322697

[28] L. Gross. Logarithmic Sobolev inequalities. Amer. J. Math., 97:1061-1083, 1975. MR-0420249

[29] Y. Hu, P. Imkeller, and M. Müller. Utility maximization in incomplete markets. Ann. Appl. Probab., 15(3):1691-1712, 2005. MR-2152241

[30] R. Jordan, D. Kinderlehrer, and F. Otto. The variational formformula of the Fokker-Planck equation. SIAM J. Math. Anal., 29(1):1-17, 1998. MR-1617171

[31] M. Kobylanski. Backward stochastic differential equations and partial differential equations with quadratic growth. Ann. Probab, 28(2):558-602, 2000. MR-1782267

[32] N. V. Krylov. Controlled Diffusion Processes. Springer, 2009. MR-2723141

[33] M. Kupper, P. Luo, and L. Tangpi. Multidimensional Markovian FBSDEs with superquadratic growth. Stoch. Proc. Appl., 129(3):902-923, 2019. MR-3913273

[34] D. Lacker. Liquidity, risk measures, and concentration of measure. Math. Oper. Res., 43(3):693-1050, 2018. MR-3846074

[35] D. Lacker, M. Shkolnikov, and J. Zhang. Inverting the Markovian projection, with an application to local stochastic volatility models. Ann. Probab., to appear, 2019.

[36] M. Ledoux. The Concentration of Measure Phenomenon, volume 89. American Mathematical Society, 2001. MR-1849347

[37] K. Marton. Bounding $\bar{d}$-distance by information divergence: a method to prove measure concentration. Ann. Probab., 24(2):857-866, 1996. MR-1404531

[38] P. Massart. Concentration inequalities and model selection. In Lecture Notes in Mathematics, volume 1896. Springer, 2007. MR-2319879

[39] D. Nualart. The Malliavin Calculus and Related Topics. Probability and its Applications (New York). Springer-Verlag, Berlin, second edition, 2006. MR-2200233

[40] M. Nutz and J. Zhang. Optimal stopping under adverse nonlinear expectation and related games. Ann. Appl. Probab., 25(5):2503-2534, 2015. MR-3375882

[41] F. Otto and C. Villani. Generalization of an inequality by Talagrand and links with the logarithmic Sobolev inequality. J. Funct. Anal., 173(2):361-400, 2000. MR-1760620

[42] S. Pal. Concentration of multidimensional diffusions and their boundary local times. Probab. Theory Relat. Fields, 154(1-2):225-254, 2012. MR-2981423

[43] S. Pal and M. Shkolnikov. Concentration of measure for Brownian particles systems interacting through their ranks. Ann. Appl. Probab., 24(4):1482-1508, 2014. MR-3211002

[44] E. Pardoux and S. Peng. Adapted solution of a backward stochastic differential equation. Systems Control Letter, 14:55-61, 1990. MR-1037747

[45] E. Pardoux and S. Peng. Backward stochastic differential equations and quasilinear parabolic partial differential equations. In B. Rozuvskii and R. Sowers, editors, Stochastic partial differential equations and their applications, volume 176, pages 200-217. Springer, Berlin, New York, 1992. MR-1176785

[46] S. Riedel. Transportation-cost inequalities for diffusions driven by Gaussian processes. Electr. J. Probab., 22(24):1-26, 2017. MR-3622894

[47] B. Saussereau. Transportation inequalities for stochastic differential equations driven by fractional Brownian motion. Bernoulli, 18(1):1-23, 2012. MR-2888696

[48] Z. Schuss. Singular perturbation methods in stochastic differential equations of mathematical Physics. SIAM Rev., 22(2):119-155, 1980. MR-0564560

[49] M. Talagrand. Transportation cost for Gaussian and other product measures. Geom. Funct. Anal., 6(3):587-600, 1996. MR-1392331

[50] L. Tangpi. Concentration of dynamic risk measures in Brownian filtrations. Stoch. Proc. Appl., 129(5):1477-1491, 2019. MR-3944773 
Functional inequalities for forward and backward diffusions

[51] A. S. Üstünel. Transportation cost inequalities for diffusions under uniform distance. In Stochastic Analysis and Related Topics, volume 22 of Proceeding in Mathematics and Statistics, pages 203-214. Springer, Berlin, Heidelberg, 2012. MR-3236093

[52] A. S. Üstünel and M. Zakai. Transformation of Measure on Wiener Space. Springer Monographs in Mathematics. Springer, 2000. MR-1736980

[53] C. Villani. Optimal Transport: Old and New, volume 338 of Grundlehren der Mathematischen Wissenschaften. Springer-Verlag, Berlin, Heidelberg, 2009. MR-2459454

[54] A. K. Zvonkin. A transformation of the phase space of a diffusion process that removes the drift. Mat. Sb. (N.S.), 93(135):129-149, 1974. MR-0336813 